\title{
The supersymmetric prediction for the muon transverse polarization in the $K^{+} \rightarrow \pi^{0} \mu^{+} \nu_{\mu}$ decay
}

\author{
Marco Fabbrichesi ${ }^{a, b}$ and Francesco Vissani ${ }^{a, c}$ \\ ${ }^{a}$ Istituto Nazionale di Fisica Nucleare, \\ Sezione di Trieste \\ ${ }^{b}$ Scuola Internazionale Superiore di Studi Avanzati, \\ Via Beirut 2-4, I-34013 Trieste, Italy \\ ${ }^{c}$ International Centre for Theoretical Physics, \\ Strada Costiera 11, I-34013 Trieste, Italy
}

\begin{abstract}
The muon transverse polarization in the $K^{+} \rightarrow \pi^{0} \mu^{+} \nu_{\mu}$ decay will be measured at the $10^{-4}$ level in forthcoming experiments. We compare the phenomenological perspectives with the theoretical predictions in supersymmetric extensions of the standard model. In the minimal extension, CP-violating phases lead to a non-zero transverse polarization, that however is too small to account for a positive experimental signal. The problems that one encounters when departing from minimal assumptions are discussed. An observable effect is possible if the hypothesis of R-parity conservation is relaxed, but only at the price of assuming a very special pattern for the R-parity breaking couplings.
\end{abstract}

IC $/ 96 / 220$

SISSA 140/96/EP

November 1996 
A non-vanishing component of the muon polarization, transverse to the decay plane of the $K^{+} \rightarrow \pi^{0} \mu^{+} \nu_{\mu}\left(K_{\mu 3}^{0}\right)$ process, would signal CP-violating effects [1], if bigger than $\mathcal{P}_{\perp} \sim 10^{-6}$ (the contribution due to final state interactions [2]). Such a signal would be particularly interesting, since it would imply physics beyond the standard model [3].

Forthcoming experiments at DA $\Phi$ NE [4], KEK [5] and BNL [6] may push the present limit $\mathcal{P}_{\perp}<5 \times 10^{-3}$ [7] by more than one order of magnitude: $\mathcal{P}_{\perp}<2.8 \times 10^{-4}$ (from [8]), or perhaps obtain a positive result. For this reason, it is important to state the prediction for this observable in all those models that are potential candidates to describe physics beyond the standard model (see [2, 9, 10, 11, 12, 13] for earlier studies).

The outline of the present work is the following. Sect. 1 is devoted to a phenomenological discussion, including the relationship between the polarization and the invariant form factors, the discussion of the experimental perspectives and the specification of the effect of strong interactions. We analyze in Sect. 2 the size of the transverse polarization in possible extensions of the standard model. We focus in particular on supersymmetric models (see [14] and [15] for previous analyses): the minimal extension of the standard model, and models with explicit R-parity breaking. The last Section is devoted to the conclusions.

\section{Decay Form Factors and Muon Polarization}

The form of the invariant amplitude suggested by the Particle Data Group is [16]:

$$
\begin{aligned}
\mathcal{M}=G_{F} \sin \theta_{C} \overline{u\left(p_{\nu}\right)_{L}}[ & \left(F_{+}\left(p_{K}+p_{\pi}\right)^{a}-F_{-}\left(p_{K}-p_{\pi}\right)^{a}\right) \gamma_{a} \\
& \left.+2 F_{S} m_{K}+2 i \frac{F_{T}}{m_{K}} \sigma_{a b} p_{K}^{a} p_{\pi}^{b}\right] v\left(p_{\mu}, \vec{s}_{\mu}\right) .
\end{aligned}
$$

The leptons in the final state are a left-handed neutrino with four-momentum $p_{\nu}$, and an antimuon with four-momentum $p_{\mu}$ and spin $\vec{s}_{\mu}$ (Notice that, due to the chirality projector, we can account for an additional contribution $\delta F_{T} / m_{K} p_{K}^{a} p_{\pi}^{b} \sigma^{c d} \epsilon_{a b c d}$ to the bracketed term simply by redefining $\left.F_{T} \rightarrow F_{T}-\delta F_{T}\right)$.

In eq. (1.1) we distinguish between the contribution of the usual $V-A$ interactions and that of other possible interactions because final state interactions [2] and the standard model sources of $\mathrm{CP}$ violation [3] give a negligible contribution to $F_{S}$ and $F_{T}$. 
The form factors $F_{+}, F_{-}, F_{S}, F_{T}$ in eq. (1.1) depend in general on the hadronic momentum transferred, $q^{2}=\left(p_{K}-p_{\pi}\right)^{2}$; CP invariance implies that they are relatively real. They can be calculated once the model and the hadronic matrix elements are specified.

\subsection{The Transverse Polarization}

By the use of the equations of motions we can recast (1.1) in the form:

$$
\mathcal{M}=2 G_{F} \sin \theta_{C} m_{K} F_{+}\left(1-\xi_{T} \frac{m_{\mu}}{m_{K}}\right) \overline{u\left(p_{\nu}\right)_{L}}\left[\frac{p_{K}^{a}}{m_{K}} \gamma_{a}+\zeta\right] v\left(p_{\mu}, \vec{s}_{\mu}\right) .
$$

The amplitude for scalar interactions is proportional to

$$
\zeta=\frac{1}{2}\left[\frac{m_{\mu}}{m_{K}}+\left(\frac{m_{\mu}}{m_{K}} \xi+2 \xi_{S}+\frac{2 p_{K} \cdot\left(p_{\nu}-p_{\mu}\right)+m_{\mu}^{2}}{m_{K}^{2}} \xi_{T}\right)\left(1-\frac{m_{\mu}}{m_{K}} \xi_{T}\right)^{-1}\right]
$$

where we defined

$$
\xi=\frac{F_{-}}{F_{+}}, \quad \xi_{S}=\frac{F_{S}}{F_{+}} \quad \text { and } \quad \xi_{T}=\frac{F_{T}}{F_{+}} .
$$

We assume that the parameters $\xi, \xi_{S}, \xi_{T}$ are constants. Actually, writing eq. (1.1) with four form factors makes sense only if their dependence on the invariants is to a certain extent specified: Lorentz invariance alone would require just two form factors.

The form (1.2) is very convenient in evaluating the decay rate (this was originally pointed out in [17). In bispinorial notations we can write:

$$
\mathcal{M} \propto \sqrt{2 E_{\nu} 2 E_{\mu}} \phi\left(-\vec{\beta}_{\nu}\right)^{\dagger}\left[b_{+}+b_{-} \vec{\beta}_{\mu} \cdot \vec{\sigma}\right] \phi\left(-\vec{s}_{\mu}\right),
$$

where $\vec{\beta}$ are the velocity vectors for the neutrino and the antimuon, and $\gamma_{\mu}=$ $1 / \sqrt{1-\beta_{\mu}^{2}}$ the antimuon Lorentz boost factor (not a gamma matrix!). The spinors $\phi(\vec{n})$ obey $\phi^{\dagger} \phi=1$ and $(\vec{n} \vec{\sigma}) \phi(\vec{n})=\phi(\vec{n})$. The two coefficients of the amplitude can be expressed as $b_{ \pm}=(\zeta \mp 1)\left(1+\gamma_{\mu}^{-1}\right)^{ \pm 1 / 2}$. All quantities are evaluated in the kaon rest frame. Squaring (1.5) we obtain:

$$
|\mathcal{M}|^{2} \propto 8 E_{\nu} E_{\mu} \mathcal{P} \cos ^{2}[\Theta / 2]
$$

where $\Theta$ is the angle between the observed polarization $\vec{s}_{\mu}$ and the vector $\overrightarrow{\mathcal{P}}$,

$$
\begin{aligned}
\overrightarrow{\mathcal{P}} & =\left[|1-\zeta|^{2} \gamma_{\mu}^{-1}-2 \operatorname{Re} \zeta\left(1-\gamma_{\mu}^{-1}\right)\right] \vec{\beta}_{\nu} \\
& +\left[1-|\zeta|^{2}+|1+\zeta|^{2} \vec{\beta}_{\nu} \cdot \vec{\beta}_{\mu}\left(1+\gamma_{\mu}^{-1}\right)\right] \vec{\beta}_{\mu} \\
& +\operatorname{Im} \zeta\left[\vec{\beta}_{\nu} \times \vec{\beta}_{\mu}\right]
\end{aligned}
$$


whose modulus, which enters formula (1.6) is:

$$
\mathcal{P}=1+\vec{\beta}_{\nu} \cdot \vec{\beta}_{\mu}-2 \operatorname{Re} \zeta \gamma_{\mu}^{-1}+|\zeta|^{2}\left(1-\vec{\beta}_{\nu} \cdot \vec{\beta}_{\mu}\right)
$$

It is worthwile emphasize some aspects of this result, which was originally derived in [18, 19]:

(a) The probability of transition, proportional to $|\mathcal{M}|^{2}$, is small for small lepton energies.

(b) The three terms in equation (1.8) have a clear interpretation: the first (last) describes left-(right-)handed particle, produced by vector (scalar) interactions, and the second is a typical interference term $(\zeta$ parameterizes the relative amount of scalar-like interactions).

(c) The squared cosine factor in (1.6) indicates that the antimuon produced in the decay is completely polarized along $\overrightarrow{\mathcal{P}}$. The non-polarized case is recovered upon averaging (according to $\cos ^{2}[\Theta / 2] \rightarrow 1 / 2$ ).

(d) For slow antimuons, formula (1.7) shows that the polarization is parallel to the neutrino velocity vector. This is clearly due to the fact that the neutrino is in a negative helicity state.

(e) The transverse polarization, the last term in (1.7), is maximum when the antimuon and the neutrino velocities are orthogonal in the laboratory frame.

\subsection{Phenomenological Remarks and Experimental Perspectives}

In the Dalitz plot distributions, the imaginary part of the parameter $\zeta$ in eq. (1.3) (or equivalently $\xi$ and/or $\xi_{S}$ and/or $\xi_{T}$ in eq. (1.4)) enters only quadratically (compare with eq. (1.8)). On the other hand, the dependence of (1.7) on $\operatorname{Im} \zeta$ is linear and therefore the transverse polarization would give to a large extent an independent information on the form factors.

Recently a simple experimental method has been proposed [8] to detect the effects of the polarizations at DA $\Phi$ NE without the need of a polarimeter. It is based on the fact that the direction of the positron emitted in the antimuon decay is correlated to $\overrightarrow{\mathcal{P}}$, and therefore to the kinematical variables of the decay that produced the antimuon. There is a different probability of emission above and below the decay plane if $\zeta$ has an imaginary part (the direction "above the decay plane" is specified by 
the vector $\beta_{\nu} \times \beta_{\mu}$; no definition is needed when the particles are collinear). Recalling that the differential probability of emission of a positron with angle $\theta$, measured from the antimuon polarization direction, is $(1+1 / 3 \cos \theta) d \Omega / 4 \pi$, where $\Omega$ is the solid angle, we find $P($ above $)-P($ below $)=\mathcal{P}_{\perp} /(6 \mathcal{P})$, where $\mathcal{P}_{\perp}=\operatorname{Im} \zeta\left|\vec{\beta}_{\nu} \times \vec{\beta}_{\mu}\right|$. Therefore the asymmetry rate is:

$$
\operatorname{Im} \zeta \frac{G_{F}^{2} \sin ^{2} \theta_{C}}{48 \pi^{3}} m_{K}\left|F_{+}\left(1-\xi_{T} \frac{m_{\mu}}{m_{K}}\right)\right|^{2}\left|\vec{p}_{\nu} \times \vec{p}_{\mu}\right| d E_{\nu} d E_{\mu}
$$

Notice that, according to eqs. (1.3) and (11.7), if the leading contribution to the transverse polarization comes from $\operatorname{Im} \xi$ or $\operatorname{Im} \xi_{S}$, then $\mathcal{P}_{\perp}$ points in a given halfspace with respect to the decay plane; on the contrary, if $\mathcal{P}_{\perp}$ is related to $\operatorname{Im} \xi_{T}$ it can point in both directions because the factor $\left(E_{\nu}-E_{\mu}+m_{\mu}^{2} / m_{K}\right)$ changes sign (see eq. (1.3)).

In this way, DA $\Phi$ NE can provide a factor ten improvement of the current limits, and therefore may reveal transverse polarization effects if [?:

$$
\operatorname{Im} \xi_{S} \quad \text { or } \quad \operatorname{Im} \xi_{T} \gtrsim 2 \times 10^{-3}
$$

It is suggestive to compare this figure with the most recent experimental analysis of $K_{e 3}^{0}$ decays [20], for which the best fit of the Dalitz-plot distributions requires $F_{S}=(7 \pm 1.6 \pm 1.6) \times 10^{-2}, F_{T}=(5.3 \pm 1.0 \pm 1.0) \times 10^{-1}$. If similar values for $K_{\mu 3}^{0}$ decay parameters are assumed, an imaginary part as small as $0.5 \%$ would lead to an observable signal.

\subsection{Current Form Factors}

The Lorentz invariant decomposition of the hadronic matrix elements introduces five form factors:

$$
\begin{aligned}
\left\langle\pi^{0}\left|\bar{s}(0) \gamma^{a} u(0)\right| K^{+}\right\rangle= & \frac{1}{\sqrt{2}}\left[f_{+}\left(q^{2}\right)\left(p_{K}+p_{\pi}\right)^{a}-f_{-}\left(q^{2}\right)\left(p_{K}-p_{\pi}\right)^{a}\right] \\
\left\langle\pi^{0}|\bar{s}(0) u(0)| K^{+}\right\rangle= & \sqrt{2} m_{K} f_{S}\left(q^{2}\right) \\
\left\langle\pi^{0}\left|\bar{s}(0) \sigma^{a b} u(0)\right| K^{+}\right\rangle= & \frac{i}{\sqrt{2} m_{K}} f_{T}\left(q^{2}\right)\left(p_{K}^{a} p_{\pi}^{b}-p_{K}^{b} p_{\pi}^{a}\right) \\
& +f_{T}^{\prime}\left(q^{2}\right) \epsilon^{a b c d}\left(p_{K}\right)_{a}\left(p_{\pi}\right)_{b}
\end{aligned}
$$

\footnotetext{
${ }^{\dagger}$ See ref. [8]. Note however that the different dependence on the energy in eq. (1.3) implied by the tensor form factor requires a different experimental analysis from the case in which one assumes a pure scalar contribution (the one considered in $[\mathbb{8}$ ).
} 
The specification of these form factors amounts to the description of the nonperturbative effects of strong interactions. Renormalization group factors induced by QCD are of order unity for such a semi-leptonic decay and we shall ignore them. At tree level, $V-A$ structure entails only the first operator. The other two are in general present when new interactions are introduced.

The flavor $S U(3)$ symmetry imposes relations on the vector form factors so that $f_{+}(0)=-1$ and $f_{-}\left(q^{2}\right)=0$ (see for instance the discussion in [21]). For the purpose of describing the transverse polarization, it is adequate to consider such a $S U(3)$ symmetric limit.

It is important to point out that the precise knowledge of the form factors, and in particular of their momentum dependence is important in testing the hypothesis of pure standard model interactions when studying the $K_{\mu 3}$ Dalitz plot distributions.

The scalar form factor can be computed considering the matrix element of the divergence of the vector current, and then using the free equations for the quark fields (as described in [9]):

$$
f_{S}=\frac{\left(m_{K}^{2}-m_{\pi}^{2}\right) f_{+}+q^{2} f_{-}}{2 m_{K}\left(m_{s}-m_{u}\right)} \simeq \frac{m_{K}}{2 m_{s}} f_{+}
$$

This estimation is subject to considerable uncertainty due to the use of the free equations of motion and the value of $m_{s}$ (we use the central value of the recent determination $m_{s} \equiv \bar{m}_{s}(1 \mathrm{GeV})=175 \pm 25 \mathrm{MeV}$ [22]). Nevertheless, it is sufficiently accurate for the purpose of estimating the muon transverse polarization.

For completeness, we also present the estimation of the tensor form factors, that has been first evaluated in [23]. In the context of the chiral quark model [24], at the leading order, we find:

$$
f_{T} \simeq \frac{6 m_{K} M}{\Lambda_{\chi}^{2}} \approx 1, \quad f_{T}^{\prime} \approx 0 .
$$

where $M$ is the constituent quark mass, a model dependent parameter $(M=220$ $\mathrm{MeV}$ in the above), and $\Lambda_{\chi} \approx 1 \mathrm{GeV}$ is the chiral symmetry breaking scale.

\section{The Theoretical Prediction}

Which kind of models can lead to a non-zero transverse polarization, and what are their phenomenological implications? This question will be discussed within the supersymmetric extensions of the standard model. We first examine the minimal 
extension, where the effect arises at the one-loop level, and then consider R-parity non-conserving interactions, that can give an effect already at the tree level.

\subsection{Minimal Supersymmetric Standard Model}

\section{Introduction}

The supersymmetrization of the standard model requires enlarging the spectrum of the theory. The squarks and the sleptons are the scalar partner of the quarks and leptons; two Higgs doublets are present, each one paired with a fermionic doublet (higgsino); similarly, for each gauge boson one fermionic degree of freedom appears (gaugino). According to the usual convention we denote the supersymmetric particles by a tilde: for instance $\tilde{g}$ is the gluino, $\tilde{q}$ a generic squark. The values of the supersymmetric partners masses depend on the mechanism of supersymmetry breaking - an open question at present.

$S U(2)$ breaking brings in an important parameter, the ratio

$$
\tan \beta=\frac{\left\langle H_{2}\right\rangle}{\left\langle H_{1}\right\rangle}
$$

which is bounded by the requirement of perturbative Yukawa couplings to be approximatively between 1 and 50. At the same time, the higgsinos and the gauginos (except the gluino) mix; the resulting mass eigenstates are called neutralinos $\left(\tilde{\chi}_{i}^{0}\right)$ and charginos $\left(\tilde{\chi}_{i}^{+}\right)$. Another effect of $S U(2)$ breaking is the mixing of the squarks which are partner of the left and the right quarks (called left and right squarks); similarly for the sleptons. We have:

$$
\begin{aligned}
& m_{\tilde{u}_{i} \mathrm{LR}}^{2}=m_{u_{i}}\left(A_{u_{i}}-\mu^{*} \cot \beta\right), \\
& m_{\tilde{d}_{i} \mathrm{LR}}^{2}=m_{d_{i}}\left(A_{d_{i}}-\mu^{*} \tan \beta\right),
\end{aligned}
$$

where $i=1,2,3$ is the generation index, $A_{u_{i}}$ and $A_{d_{i}}$ are parameters of the Higgssquark-squark, soft-supersymmetry-breaking interactions and $\mu$ is a supersymmetryconserving mass parameter. These same parameters enter also the interaction vertices of the Higgs with the squarks, as we will see below in a noticeable case.

The massive supersymmetric parameters can be complex; in particular phases can be present in the gaugino masses, in the $A$ - and $\mu$-parameters defined abovet. These

\footnotetext{
${ }^{\dagger}$ In the constrained version called low-energy supergravity model there may be at most two new phases.
} 
phases lead to a muon transverse polarization even if there is no new flavor violation that is related to the supersymmetric parameters; our study extends the analysis of [14] to the region in which $\tan \beta$ is large. Then we will comment on the effect of relaxing the minimal hypothesis on supersymmetric flavor violation; the importance of this point has been stressed in [15].

\section{The transverse polarization}

To evaluate the possibility to have a positive experimental signal, we need an estimation of the muon transverse polarization. In the following an upper bound on this effect is given. It shows that the transverse polarization is too small to be detected.

For this sake, let us consider the gluino exchange diagram in Fig. 1(a). The new supersymmetric phases are present in the gluino-squark loop, which induces the effective coupling between the $u$ - and $s$-quarks and the charged Higgs field $\mathrm{H}^{-}$:

$$
\frac{g_{s}^{2}}{(4 \pi)^{2}}\left(V_{u s} \frac{m_{s}}{v} A_{s} \tan \beta\right) \frac{m_{\tilde{g}}}{m_{\tilde{q}}^{2}} \cdot\left(\bar{s}(x) P_{L} u(x)\right) H^{-}(x),
$$

where $V$ is the Cabibbo-Kobayashi-Maskawa matrix, $g_{s}$ the strong coupling and $v=$ $174 \mathrm{GeV}$. In formula (2.3) we only kept the part of the $H^{-}-\tilde{q}$ coupling that grows with $\tan \beta$; the gluino mass $m_{\tilde{g}}$ provides the chirality flip, and the squark mass $m_{\tilde{q}}$ gives the correct dimension. The exchange of charged Higgs $H^{-}$therefore lead to the effective operator:

$$
\sin \theta_{C} G_{S} \cdot\left(\bar{s}(x) P_{L} u(x)\right)\left(\bar{\nu}_{\mu}(x) P_{R} \mu(x)\right),
$$

where, using (1.14):

$$
G_{S}=\frac{1}{m_{H^{-}}^{2}} \frac{g_{s}^{2}}{(4 \pi)^{2}} \frac{m_{\mu} m_{s}}{v^{2}} \frac{A_{s} m_{\tilde{g}}}{m_{\tilde{q}}^{2}} \tan ^{2} \beta .
$$

The coupling of the charged Higgs with the lepton has brought in a second factor $\tan \beta$, and this is the origin of the enhancement of this type of diagram.

Accordingly to previous discussion we find:

$$
F_{S}=\frac{G_{S}}{2 \sqrt{2} G_{F}} \frac{f_{S}}{f_{+}} \sim 6 \times 10^{-5}\left(\frac{A_{s} m_{\tilde{g}}}{m_{\tilde{q}}^{2}}\right)\left(\frac{100 \mathrm{GeV}}{m_{H^{-}}}\right)^{2}\left(\frac{\tan \beta}{50}\right)^{2} .
$$

This estimation shows that non-zero contributions to the form factor $F_{S}$ are possible in the minimal supersymmetric extension of the standard model if the supersymmetric 
parameters $A_{s}$ or $m_{\tilde{g}}$ are complex. However, it also shows why it is difficult to expect a positive signal in the next generation searches of transverse polarization. In fact, in the numerical estimation (2.6), the transverse polarization can attain relatively large values only if we assume:

(a) quite light supersymmetric masses;

(b) large values of $\tan \beta$;

(c) large supersymmetric phases (in the gluino masses and $A_{s}$ ).

We recall two possible unpleasant features of the large $\tan \beta$ scenario: first, it usually implies fine-tuning in the parameters of the scalar sector; secondly, amplitudes depending on the Yukawa parameters (like those for "dimension five" proton decay, or those for $b \rightarrow s \gamma$ transition) may become too large in this limiting case. (Notice, for the following discussion, that $m_{\tilde{b}_{\mathrm{LR}}}^{2}$ defined in eq. (2.2) is expected to be large, of the order of $m_{b} \times \mu \tan \beta$ : cancellations with the $A_{b}$-term contribution would imply color-breaking-minima in the scalar potential.) Furthermore, let us remark that the parameter $A_{s}$ is not free from experimental constraints. In fact the gluino $s$-quark loop will generate an electric dipole moment for the $s$-quark; this effect is further amplified by a $\tan \beta$ factor (present in the left-right $s$-squarks mixing).

Let us consider now the diagram in Fig. 1(b), that is obtained from Fig. 1(a) by replacing $u$ with $\nu, s$ with $\mu$, and $\tilde{g}$ with $\tilde{Z}$. The loop is at the leptonic end, and the phases now appear in the soft-breaking leptonic parameter $A_{\mu}$ and in the zino mass. The contribution of this diagram has the same quadratic behavior in $\tan \beta$ discussed in (2.5). The amplitude is few times smaller, due to the weak gauge coupling replacing the strong one. However, the limits from muon electric dipole moment are much weaker. For this reason, the leading effect might be related to neutralino exchange diagrams.

In view of the negative result we will not proceed in the discussion, and regard (2.6) as an upper bound on the effect. Such an upper bound is rather robust in the sense that the final number is small, no matter what loop diagram we consider.

\section{On Supersymmetric Flavor Violations and Transverse Polarization}

Recently, a supersymmetric scenario which makes room for observable effects in the next generation of transverse polarization experiments has been proposed [15]. 
The scenario relies on the sources of flavor violation that are provided by the supersymmetric parameters: this leads to the possibility that the gluino couplings with the $u$ - and the $s$-quarks involve the third family squarks, those with the largest couplings with the charged Higgs (compare with eq. (2.3)). Three major assumptions have to be fulfilled:

(a) $V_{32}^{D_{L}}$, that quantifies the mixing of the "left" squark $\tilde{s}_{L}$ with $b_{L}$ in the gluino coupling, and $V_{31}^{U_{R}}$, analogously defined, are order unity and carry large phases;

(b) the masses of the gluino and of the squarks are close to their present experimental value;

(c) $\tan \beta$ is large.

Unfortunately, this set of assumptions becomes problematic as soon as we consider the rate of the $b \rightarrow s \gamma$ transition, that is known to be fairly well reproduced by the standard model amplitude alone.

To make this point explicit, let us consider the gluino-bottom-squark diagram for this transition. The gluino mass provides the chirality flip, and one $m_{\tilde{b}_{\mathrm{LR}}}^{2}$-insertion in the bottom-squark line allows one to construct the dipole operator. Let us compare this contribution with that of the standard model:

$$
\frac{\mathcal{M}_{\tilde{g}}}{\mathcal{M}_{W}}=\frac{\frac{e \alpha_{s}}{4 \pi} \frac{m_{\tilde{g}}}{m_{\tilde{\tilde{c}}}^{2}}\left(V_{32}^{D_{L}} \frac{m_{\tilde{b} \mathrm{~L} R}^{2}}{m_{\tilde{b}}^{2}} V_{33}^{D_{R}}\right) \mathcal{F}_{\tilde{g}}\left(\frac{m_{\tilde{b}}^{2}}{m_{\tilde{g}}^{2}}\right)}{\frac{e \alpha_{W}}{4 \pi} \frac{m_{b}}{m_{W}^{2}}\left(V_{t s} V_{t b}\right) \mathcal{F}_{W}\left(\frac{m_{t}^{2}}{m_{W}^{2}}\right)} .
$$

The loop functions $\mathcal{F}$ were computed in [25]; assuming, consistently with [15], that $m_{\tilde{b}} \sim m_{\tilde{g}}$, the functions amount to a factor close to unity. From eq. (2.7) we come to the estimate:

$$
\frac{\mathcal{M}_{\tilde{g}}}{\mathcal{M}_{W}} \sim 10^{3} \times\left(\frac{V_{32}^{D_{L}}}{1 / \sqrt{2}}\right)\left(\frac{V_{33}^{D_{R}}}{1 / \sqrt{2}}\right)\left(\frac{\tan \beta}{50}\right)\left(\frac{\mu m_{\tilde{g}} m_{W}^{2}}{m_{\tilde{b}}^{4}}\right)
$$

where we assumed $V_{t s} \sim V_{c b}$. Therefore this contribution may trigger a $b \rightarrow s \gamma$ transition one million time faster then that of the standard model.

The way out suggested in [15] is that the chargino contribution, whose importance has been emphasized in the literature [26], cancels the gluino amplitude and accordingly makes the bounds discussed above less stringent. It is however hard to justify such a precise cancellation, since different parameters enter the two amplitudes. Moreover, it is not clear if it is possible to implement such a cancellation 
without suppressing the transverse polarization effect as well. Finally, in a purely phenomenological scenario, like the one considered, one would expect the dominance of the gluino exchange amplitude over all the other ones, chargino exchange included, since no smallness factors are attached to the gluino couplingst.

In conclusion, we feel that it is difficult to account for a transverse polarization within the context of the minimal supersymmetric extension of the standard model even after making allowence for family mixing and flavor violations.

\subsection{R-Parity Breaking Models}

\section{Introduction}

The requirement of gauge invariance permits the following renormalizable interactions in the superpotential:

$$
\begin{aligned}
& \left(Y_{j k}^{E} H_{1}+\lambda_{i j k} L_{i}\right) L_{j} E_{k}^{c}+\left(Y_{j k}^{D} H_{1}+\lambda_{i j k}^{\prime} L_{i}\right) Q_{j} D_{k}^{c} \\
& +\left(\mu H_{1}+\mu_{i} L_{i}\right) H_{2}-Y_{j k}^{U} H_{2} Q_{j} U_{k}^{c}+\lambda_{i j k}^{\prime \prime} D_{i}^{c} D_{j}^{c} U_{k}^{c}
\end{aligned}
$$

Besides the interactions of quark $\left(Q, U^{c}, D^{c}\right)$ and lepton $\left(L, E^{c}\right)$ with Higgs $\left(H_{1}, H_{2}\right)$ superfields, and the $\mu$-term, we have the $R$-parity breaking interactions, parameterized by $\lambda, \lambda^{\prime}, \lambda^{\prime \prime}$ and $\mu_{i}$, that have no correspondence in the standard model lagrangian, and break either the lepton $\left(\lambda, \lambda^{\prime}, \mu_{i}\right)$ or the baryon $\left(\lambda^{\prime \prime}\right)$ number. We consider strict baryon number conservation $\left(\lambda^{\prime \prime}=0\right)$ in the following, in order to avoid strong matter stability bounds 27, 28.

Since the R-parity breaking couplings are a-priori complex quantities, as remarked in [29], it is important to ask whether they can manifest themselves in a large $\mathcal{P}_{\perp}$. Before answering this question we must recall some relevant information on the model under consideration.

Let us assume a generic pattern of the R-parity breaking couplings $\lambda, \lambda^{\prime}$, and $\mu_{i}$. We fix the basis in the $H_{1}, L_{i}$ four-dimensional space in two steps:

(1) we redefine the Higgs superfield in such a way that $\mu_{i}$ terms are absent;

(2) we further rotate the three lepton superfields in order to make the lepton mass matrix diagonal (analogously for the quark superfields).

\footnotetext{
${ }^{\dagger}$ A different situation happens in the models where flavor-changing gluino couplings are induced radiatively by the usual Yukawa couplings, and therefore are strongly suppressed - see for instance 25.
} 
In general, R-parity breaking interactions of scalars induce vacuum expectation values of sneutrino fields $\tilde{\nu}_{i}$ 30, 31. In fact the similarity between the scalar leptons and the usual Higgs doublets is almost complete in the model under consideration.

Supersymmetry manifests itself by providing relations among the various interactions; for instance, $\lambda_{i j k}^{\prime} L_{i} Q_{j} D_{k}^{c}$ describes at the same time the interactions of quarks with the slepton $\tilde{l}_{i}(x)$ (that in this context can be thought of as an Higgs doublet) and the interactions in which the squarks behave as leptoquarks. This implies that this model is more predictive, and more constrained, than a multiHiggs (or a leptoquark) model.

The Yukawa $Y^{D}, Y^{E}$ interactions are fixed by the tree level condition:

$$
\begin{aligned}
Y_{j k}^{D} & =\frac{M_{j k}^{D}}{\left\langle H_{1}\right\rangle}-\lambda_{i j k}^{\prime} \frac{\left\langle\tilde{\nu}_{i}\right\rangle}{\left\langle H_{1}\right\rangle} \\
Y_{j k}^{E} & =\frac{M_{j k}^{E}}{\left\langle H_{1}\right\rangle}-2 \lambda_{i j k} \frac{\left\langle\tilde{\nu}_{i}\right\rangle}{\left\langle H_{1}\right\rangle}
\end{aligned}
$$

to reproduce the observed fermion masses. The $\lambda$ and $\lambda^{\prime}$ couplings are also subject to experimental bounds from of various processes [27, 32]. In particular the couplings that trigger flavor-changing neutral current transitions are quite strongly constrained.

Neutrino masses can be induced in this model by two mechanisms: due to the mixing of neutrinos and zinos, caused by the sneutrino vacuum expectation values, but also by fermion-scalar loops with two R-parity breaking vertices [30]. We will be interested in this second type of contribution in the following. Let us therefore recall that the coupling $\lambda_{i j j}^{\prime}$ gives:

$$
\left(\delta m_{\nu_{i}}\right)^{\text {loop }} \sim \frac{3}{8 \pi^{2}} \frac{\left(\lambda_{i j j}^{\prime}\right)^{2}}{m_{\tilde{d}_{j}}^{2}} m_{d_{j}} m_{\tilde{d}_{j} \mathrm{LR}}^{2}
$$

where the factor 3 is for color; this factor is absent for loops induced by $\lambda$ couplings. A glance at eq. (2.2) shows that there are two fermion mass insertions in (2.11): this must be so, since the gauge invariant operator for neutrino mass is of the form neutrino-neutrino-Higgs-Higgs, and the Higgs field is coupled to fermion masses.

\section{The transverse polarization}

Let us assume that the couplings in the interactions:

$$
\lambda_{322}^{\prime} L_{3} Q_{2} D_{2}^{c} \quad \text { and } \quad \lambda_{322} L_{3} L_{2} E_{2}^{c}
$$

in (2.9) are not small, whereas the other R-parity breaking couplings are suppressed to obey the experimental bounds. Let us further assume that the quark doublet is 
$Q=\left(V^{\dagger} U, D\right)$, in order to avoid sneutrino mediated flavor-changing interactions. The couplings in eq. (2.12) induce, after integrating away the slepton $\tilde{e}_{3}$, the effective operator:

$$
\sin \theta_{C} G_{S} \cdot\left(\bar{s}(x) P_{L} u(x)\right)\left(\bar{\nu}_{\mu}(x) P_{R} \mu(x)\right)
$$

where

$$
G_{S}=\frac{\lambda_{322}^{\prime} \lambda_{322}}{m_{\tilde{e}_{3}}^{2}}
$$

This yields the scalar form factors:

$$
F_{S}=\frac{G_{S}}{2 \sqrt{2} G_{F}} \frac{f_{S}}{f_{+}} \sim 4 \times 10^{-2}\left(\frac{\lambda_{322^{\prime}}}{0.1}\right)\left(\frac{\lambda_{322}}{0.1}\right)\left(\frac{100 \mathrm{GeV}}{m_{\tilde{e}_{3}}}\right)^{2} .
$$

Comparing with eqs. (1.4) and (1.10), we conclude that a phase larger than 1/20 would lead to a positive signal in the next generation searches of transverse polarization

The couplings above are however subject to bounds due to the neutrino masses. Using the estimate in eq. (2.11), and asking the tau neutrino mass to be $10 \mathrm{eV}$, we find an upper limit on the couplings considered: $\lambda_{322} \lesssim 0.02$ and $\lambda_{322}^{\prime} \lesssim 0.03$, where the supersymmetric massive parameters have been assumed around $100 \mathrm{GeV}$. This implies:

$$
\operatorname{Im} \xi_{S} \lesssim 2 \times 10^{-3}
$$

that, with a phase of order unity could still give an observable transverse polarization effect. On the contrary, if the scale of supersymmetry breaking would be one order of magnitude larger, around the $\mathrm{TeV}$, it would be improbable to have a detectable effect. Let us notice that a neutrino in the $10 \mathrm{eV}$ range is a hot dark matter candidate, and therefore well motivated. There are several possibilities that allow one to relax the bound (2.16) (but, to advocate one or more of them, would take us beyond the present phenomenological approach). One can:

(1) assume a cancellation in the left-right mixing in eq. (2.11) to diminish the induced neutrino mass;

(2) allow for compensation between the $\lambda$ and $\lambda^{\prime}$ contributions, or else partial cancellations of the loop-induced and the sneutrino-vacuum expectation value contributions to $m_{\nu_{\tau}}$; 
(3) or, finally, impose the weaker bound coming from experimental studies of tau decays, $m_{\nu_{\tau}} \lesssim 20 \mathrm{MeV}$.

Last possibility can be regarded with favor, since it does not rely on cancellations. Accepting this option, one should however bear in mind that a neutrino heavier than approximatively $100 \mathrm{eV}$ has to be unstable to avoid cosmological bounds. In view of the above mentioned difficulties, and also of the very specific choice of couplings in eq. (2.12), we conclude that there is only a marginal possibility that an observable transverse polarization is related to this kind of models.

\subsection{Other Models}

Models that can generate a sizeable transverse polarization has been discussed in the literature. In particular, models including leptoquarks or new Higgs particles (more in general: new scalars coupled to fermions) [2, 9, 10, 11, 12, 13], or also fundamental tensor particles [23] has been considered.

Models with new scalars are probably the most promising candidates to account for a positive signal. In fact, complex part of the form factor of the order of:

$$
\operatorname{Im} \xi_{S} \sim 8 \times 10^{-3}
$$

can give rise to a measurable muon transverse polarization without conflicting with other observables.

\section{Conclusions}

The knowledge of the form factors in the $K_{\mu 3}^{0}$ process could give important information on CP-violation. The experimental signature is provided by the transverse polarization of the muon.

At the level of a purely phenomenological analysis, we stressed the complementarity of this experimental information with that from the analysis of the Dalitz-plot distribution; we also pointed out the importance of distinguishing between scalar and tensor form factors.

The discussion of realistic models was focused on the supersymmetric extensions of the standard model. In the minimal model, assuming that the Cabibbo-KobayashiMaskawa matrix is the only source of flavor violation, the contribution to the muon polarization is too small, even under optimistic assumptions, as in (2.6). We com-

mented on the scenario in which large supersymmetric $\mathrm{CP}$ and flavor violations are 
allowed, and showed the difficulties that arise of reconciling the assumptions required to have a large $\mathcal{P}_{\perp}$ and the observed rate of the $b \rightarrow s \gamma$ transition. Observable effects are in principle possible departing from the hypothesis of R-parity conservation, but constraints from the neutrino masses severely restricts the region of parameter space in which this can happen.

In conclusion, assuming that the minimal supersymmetric standard model (possibly with R-parity breaking interactions) is correct, we expect that future search of transverse polarization in $K_{\mu 3}^{0}$ decay should give a null result. On the other hand, a signal of transverse polarization in forthcoming experiments would point to physics different from the standard model and from its straightforward supersymmetric extensions.

\section{ACKNOWLEDGEMENTS}

The authors would like to thank S. Bertolini and E. Christova for discussions. F. V. acknowledges conversations with M. Chizhov, M.A. Diaz, N. Paver, A. Rašin, G. Senjanović and A.Yu. Smirnov. 


\section{REFERENCES}

[1] J.J. Sakurai, Phys. Rev. 109 (1958) 980.

[2] A.R. Zhitnitskii, Yad. Fiz. 31 (1980) 1024 [Sov. J. Nucl. Phys. 31 (1980) 529].

[3] E. Golowich and G. Valencia, Phys. Rev. D 40 (1980) 112.

[4] "The DAథNE Handbook", eds. L. Maiani, G. Pancheri, N. Paver, Frascati 1992.

[5] Y. Kuno, Nucl. Phys. B (Proc. Suppl.) 37A (1994) 87.

[6] R. Adair et al., "Muon Polarization Working Group Report", hep-ex/9608015.

[7] S.R. Blatt et al., Phys. Rev. D 27 (1983) 1056.

[8] P. Privitera, "Measurement of Muon Polarization in $K^{+} \rightarrow \pi^{0} \mu^{+} \nu_{\mu}$ at a $\phi$ Factory", hep-ph/9605416.

[9] H.Y. Cheng, Phys. Rev. D 26 (1982) 143.

[10] M. Leurer, Phys. Rev. Lett. 62 (1989) 1967.

[11] P. Castoldi, J.-M. Frère and G.L. Kane, Phys. Rev. D 39 (1989) 2633.

[12] R. Garisto and G. Kane, Phys. Rev. D 44 (1991) 2038.

[13] G. Bèlanger and C.Q. Geng, Phys. Rev. D 44 (1991) 2789.

[14] E. Christova and M. Fabbrichesi, Phys. Lett. B 315 (1993) 113.

[15] G.-H. Wu and J.N. Ng, "Supersymmetric Time Reversal Violation in Semileptonic Decays of Charged Mesons", hep-ph/9609314.

[16] Particle Data Group, Phys. Rev. D 54 (1996) 1;

idem, Phys. Lett. B 111 (1982) 73.

[17] L.B. Okun and I.B. Kriplovich, Yad. Fiz. 6 (1967) 821 [Sov. J. Nucl. Phys. 6 (1968) 598].

[18] S.W. MacDowell, Nuovo Cim. 9 (1958) 258. 
[19] N. Cabibbo and A. Maksymowich, Phys. Lett. 9 (1964) 352; Erratum ibidem 11 (1964) 360; ibidem 14 (1966) 72.

[20] S.A. Akimenko et al., Phys.Lett. B259 (1991) 225.

[21] "Leptons and Quarks", L.B. Okun, Amsterdam 1982, North-holland.

[22] J. Bijnens, J. Prades and E. de Rafael, Phys. Lett. B 348 (1995) 226.

[23] M. Chizhov, Mod. Phys. Lett. A 8 (1993) 2753.

A subsequent work, Phys. Lett. B 381 (1996) 359 is specifically dedicated to the study of some aspects of tensor interactions at DA $\Phi$ NE.

[24] K. Nishijima, Nuovo Cim. 11 (1959) 698;

F. Gursey, Nuovo Cim. 16 (1960) 230 and Ann. Phys. (NY) 12 (1961) 91;

J.A. Cronin, Phys. Rev. 161 (1967) 1483;

S. Weinberg, Physica 96A (1979) 327;

A. Manohar and H. Georgi, Nucl. Phys. B 234 (1984) 189;

A. Manohar and G. Moore, Nucl. Phys. B 243 (1984) 55;

D. Espriu, E. de Rafael and J. Taron, Nucl. Phys. B 345 (1990) 22.

[25] S. Bertolini, F. Borzumati, A. Masiero and G. Ridolfi, Nucl. Phys. B 353 (1991) 591.

[26] R. Barbieri and G. F. Giudice, Phys. Lett. B 309 (1993) 86, discussion of the cancellation in the supersymmetric limit;

J. Lopez, D. Nanopoulos and G. Park, Phys. Rev. D 48 (1993) 974, dependence on the sign of $\mu$;

N. Oshimo, Nucl. Phys. B 404 (1993) 20 and M. Diaz, Phys. Lett. B 322 (1994) 207, dependence on $\tan \beta$;

Y. Okada, Phys. Lett. B 315 (1993) 119, dependence on the stop splitting;

F. Borzumati, Z. Physik C 63 (1994) 291 and P. Nath and R. Arnowitt, Phys.

Lett. B 336 (1994) 395, discussion of the cancellation based on a numerical analysis of the rate;

R. Garisto and J.N. Ng, Phys. Lett. B 315 (1993) 372 and S. Bertolini and F. Vissani, Z. Physik C 67 (1995) 513, discussion based on the analytical form of the chargino amplitude.

[27] C.E. Carlson, P. Roy and M. Sher, Phys. Lett. B 357 (1995) 99. 
[28] A.Yu. Smirnov and F. Vissani, Phys. Lett. B 380 (1996) 317.

[29] C. Liu, Int. J. Mod. Phys. A 11 (1996) 4307.

[30] L.J. Hall and M. Suzuki, Nucl. Phys. B 231 (1984) 419.

[31] I-H. Lee, Phys. Lett. B 138 (1984) 121; Nucl. Phys. B 246 (1984) 120.

[32] V. Barger, G.F. Giudice and T. Han, Phys. Rev. D 40 (1989) 2987;

K. Agashe and M. Graesser, Phys. Rev. D 54 (1996) 4445;

F. Vissani, "R-Parity Breaking Phenomenology", hep-ph/9602395;

D. Choudhuri and P. Roy, Phys. Lett. B 378 (1996) 153;

G. Bhattacharyya, "R-Parity Violating Supersymmetric Yukawa Couplings: A Minireview", hep-ph/9608415. 


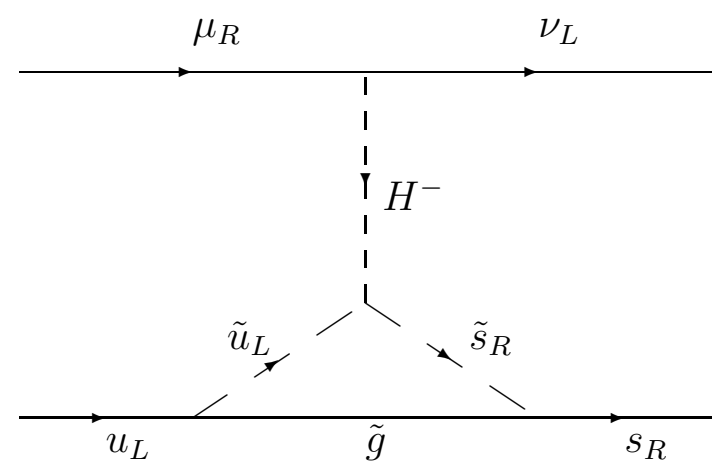

(a)

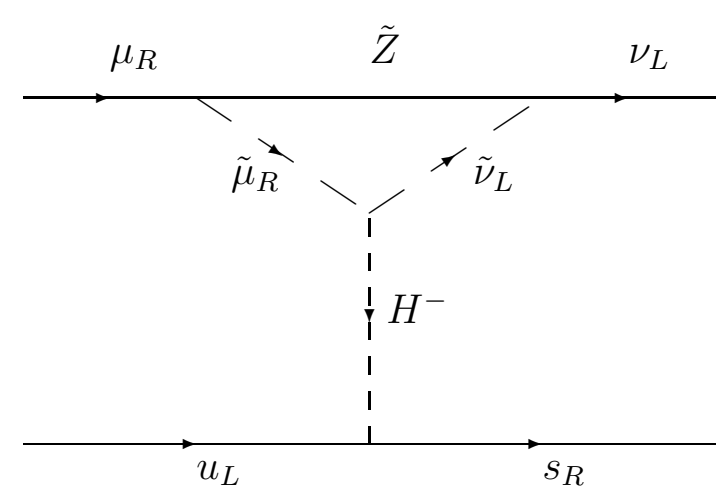

(b)

Figure 1: Two Feynman diagrams inducing scalar-type four-fermion interactions in the minimal supersymmetric extension of the standard model. 